\title{
Diode Device
}

National Cancer Institute

\section{Source}

National Cancer Institute. Diode Device. NCI Thesaurus. Code C49922.

An electronic component designed to pass current in one direction only. 